\title{
A REVIEW ON SOLID DISPERSIONS
}

\author{
KUMARI P AND DUA JS*
}

Department of pharmaceutics, Shivalik College of Pharmacy Nangal 140126 India

*Corresponding Author: Dr. Jagdeep Singh Dua: E Mail: jsdua2002@yahoo.com; Tel:

+919417220859

Received 12 ${ }^{\text {th }}$ May 2020; Revised $7^{\text {th }}$ June 2020; Accepted $13^{\text {th }}$ July 2020; Available online $1^{\text {st }}$ March 2021

\section{https://doi.org/10.31032/IJBPAS/2021/10.3.5397}

ABSTRACT

Many of the newly discovered moieties of chemical drugs are poorly soluble in water. Similarly, the classification of BCS, Class II, and IV medicines is considered to be poorly soluble in water. Hence the enhancement of oral absorption and the bioavailability of solid dosage types remains a concern to formulate scientists based on their criteria for solubility. Consequently, several techniques are being investigated to improve its solubility. One of the most powerful ways to improve solubility is strong dispersion. Solid dispersions have drawn significant interest in increasing the rate of dissolution and thus the bioavailability of a variety of hydrophobic drugs as an efficient means. Similar to conventional formulations like tablets or capsules, there are many benefits of solid dispersions that can be formulated using different methods. In this review article, we discuss some modern and innovative Solid Dispersion strategies to boost the bioavailability of several poorly soluble drugs.

\section{Keywords: Solid Dispersions, Dissolution, Bioavailability}

\section{INTRODUCTION}

The solubility / dissolution activity of a drug is an important determinant of its oral bioavailability, with the most recent frequency being the rate-limiting step towards gastrointestinal drug absorption [13]. As a consequence, poor solubility results in low bioavailability, increased dosage, large inters and intra-subject variation, and significant variations in blood drug concentrations under fed versus fasted conditions. Improving the oral bioavailability of low water-soluble drugs remains one of the most challenging aspects of drug development. The 
techniques / approaches commonly used to resolve the inconveniences associated with low water-soluble drugs typically include micronization, salt formulation, surfactant use, and drug usage $[4,5]$. All of these strategies have drawbacks too. Micronization has many drawbacks, the main one being the restricted ability to control important final particle characters such as scale, shape, composition, surface properties and electrostatic charging. Micronisation is therefore a high-energy process that causes disruptions in the drug's crystal lattice, leading to disordered or amorphous regions in the end product. The amorphous regions are thermally unstable and are thus susceptible to recrystallization after storage, particularly under hot regions $\&$ humid conditions [6, 7]. Salt formation doesn't suit all poorly water-soluble drugs to increase their solubility. The rate of dissolution of a particular salt usually varies from that of the parent compound. Strong acid sodium and potassium salts however dissolve faster than free salts. Potential salt-type disadvantages include high reactivity with ambient carbon dioxide and water leading to mud-soluble low precipitation content, epigastric discomfort due to high alkalinity. Although the use of co-solvent to improve disintegration levels poses problems such as patient enforcement and trade $[8,9]$.
Solid dispersion (SD) technique has been widely used to improve the rate of dissolution, solubility and oral absorption of drugs that are poorly soluble in water [10]. The term solid dispersions is used to characterize a class of dosage forms by which the drug is delivered in a biologically inert matrix, usually to improve bioavailability in the oral setting.

\section{MERITS OF SOLID DISPERSION}

- Increased wettability during strong dispersion contributes to greater solubility. The carriers here play an important role in improving the wettability of solid particles.

- The particles have a high degree of porosity during solid dispersion process. Increased porosity affects the carrier properties and raises the profile of drug release [11].

- Amorphous drug conditions lead to a increase in drug releases. Drugs are presented as super-saturated solutions by using a strong dispersion system and are considered a metastable polymorphism process.

- Reducing particle size in solid dispersion contributes to an increase in surface area leading to an increase in dissolution rate [12].

\section{DEMERITS OF SOLID DISPERSION}

- Vehicle Stability and Drug Problem.

- The physicochemical properties are reproducible. 
- The process of preparation is very onerous.

- Large dispersions in dosage form are difficult to prepare [13]

\section{SOLUBILITY}

STRATEGIES

DISPERSIONS

Several investigation techniques include fusion (melting), solvent evaporation, lyophilization (freeze drying), melt agglomeration process, extrusion process, spray drying technology, surfactant application, electro-static spinning device and super-critical fluid technology.

\section{1] FUSION METHOD}

Technically, the process of fusion is the less complicated method of dispersion preparation provided that the substance and carrier in the molten state are miscible. This process uses melting of the substance and carrier mixture in a metal vessel heated in an oil bath, the sample is poured on a metal plate that is placed in an ice bath immediately after fusion. Changes to the cycle involve drier water congealing to the surface of cold metal. Decomposition can be halted, affecting the fusion time and the cooling rate $[14,15]$. Another addition to the above process in which troglitazonepolyvinyl pyrrolidone (PVP) k 30 SD(s) have been found to be prepared by a closed melting point cycle. This method involves controlled mixing of water content to particular mixtures of troglitazone PVP k30 by storage in the equilibrium (adsorption system) at various levels of relative humidity or directly adding water (charging cycle) and then heating the mixer up. This method is reported to generate SD with 0 per cent apparent crystallinity [16].

Nevertheless, the fusion process does not need an organic solvent because the melting of sparingly water-soluble drug and water-soluble polymer requires a cooling step and a strong pulverizing step, requiring time-consuming multi-stage intervention. This problem was established in order to overcome method [17] for conceptualizing SD formulation as a solid-to-solid interaction between a sparsely watersoluble product, nilvadipine, and watersoluble polymer that, unlike conventional production methods, it consists of mixing a sparsely water-soluble drug and watersoluble polymer together under no more than the usual heating force in the temperature-free region, instead of heating the system to such an extent that the two materials are molten, the water-soluble component can be rendered amorphous that no previously recorded dry operation has ever been carried out.

\section{2] SOLVENT \\ EVAPORATION} METHOD:

The solvent dependent method uses the organic solvent to dissolve and closely disperse the product and the carrier molecule. Typically large amounts of 
solvents are required which can cause toxicological problems. Several researchers used the technique of solvent evaporation to check meloxicam, naproxen, rofecoxib and felodipine SD [18].

\section{3] HOT MELT EXTRUSION}

\section{METHOD:}

The extrusion is generated via Hot-stage extrusion (HME). At high drug and carrier rotational rates the suspension is removed at melting temperature for a limited period of time, previously combined. After cooling down, the resultant product is then collected and milled at room temperature. Combining hot-stage extrusion with the use of carbon dioxide as a plasticizer will bring about a reduction in processing temperature that extends the application of hot-stage extrusion to thermally labile compounds.HME also offers many advantages over conventional pharmaceutical processing techniques including lack of solvents, few processing steps, continuous activity, increased possibility of heavy dispersion and increased bioavailability [19].

\section{4] MELTING SOLVENT METHOD}

In an suitable liquid solvent, a substance is first dissolved and this solution is then added into the melting of polyethylene glycol, which can be obtained below $70^{\circ} \mathrm{C}$ without removing the liquid. The chosen solvent or the dissolved substance with the polyethylene glycol melt might not be miscible. The liquid solvent used may also influence the polymorphic form of the precipitated substance in solid dispersion [20].

\section{5] LYOPHILLISATION TECHNIQUE}

Freeze-drying requires the transfer of heat and mass to and from the preparing product. This method had been suggested as an alternative to solvent evaporation. Lyophilization was developed as a molecular mixing method in which the substance and carrier are co-dissolved, frozen and sublimated in a particular solvent to achieve lyophilized molecular dispersal.

\section{6] MELT AGGLOMERATION TECHNIQUE}

For this procedure binders are used as transport carriers. There are two types of strong dispersal formulations, the first is that the substance is sprayed on melted binder plus excipients. It is the melting of binder drug and excipients above the melting binders used. For the use of high binder material, rotary method for regulating temperature may be preferred. In product homogeneous mixing this approach is successful but greater particle size causes mass adhesion to densification and fines.

\section{7] SPRAY DRYING}

The processing of milk powder was one of the first uses of spray drying when the process was produced in the year 1920.Spray drying today finds great utility 
in the pharmaceutical industry due to the rapid drying and the specific characteristics such as particle size and final product shape. What's more, because it's 30-50 times cheaper than freezing, it's simple and cost efficient. It is a known process which is initiated by the atomisation of suspensions or droplets of fine solutions followed by a period of drying, resulting in firm particles. The process allows both fine, dust free powder and agglomerated powder to be produced with precision requirements. The operating conditions and dryer design are based on the drying properties of the product and involve specific powders [2123]. This technique prepared loperamide and peg $6000 \mathrm{SD}(\mathrm{s})$, in which solutions containing different amounts of loperamide are sprayed in relation to the total volume of solid. At $400 \mathrm{C}$, the dispersions were dried under vacuum, after spray drying until constant weight. Dichloromethane has been used to treat solvents. The prepared $\mathrm{SD}(\mathrm{s})$ showed a higher dissolution rate than pure crystalline [24] Chouhan et al [25] tested the appropriateness of this process for glibenclamide-polyglcolized glyceride $\mathrm{SD}(\mathrm{s})$ preparation. This work highlighted the progress of the levels of solubility and dissolution, as well as the improvement of the therapeutic efficacy of amorphous glibenclamide in $\mathrm{SD}(\mathrm{s})$. Some other investigators [26, 27] have reported improvement in solubility and dissolution levels. The widespread use of the organic solvent in spray drying poses problems such as product residues, environmental mental pollution and organizational safety, as well as corporate concerns such as infrastructure investment. Tanno et al [28] described a method for developing watersoluble drugs with water-soluble polymer dispersion and/or water-soluble polymer solution and a plasticizer solution using a 4-nozzle spray device.

The spray drying technique is a valuable method for obtaining spherical particles and for narrow distribution. In formulating types of solid dosages, the function of porous materials such as calcium silicate, controlled porous glass, and porous cellulose is appreciated since they impart unique characteristics such as reduced melting point and decreased chemical crystallinity in pores. Furthermore, under extreme storage-age conditions, porous materials monitor meta-stable crystals in $\mathrm{SD}(\mathrm{s})$ with polymorphs and stabilizes. Additionally, porous silica has been documented to increase the solubility and dissolution of in- domethacin and tolbutamide $[29,30]$.

\section{8] DROPPING METHOD}

This technique, invented by Ulrich et al to facilitate the crystallization of various chemicals, can overcome some of the difficulties inherent in the other approach, is a modern method of producing round 
particles from melted solid dispersions. Pipette is a continuous dispersion of a liquid mixture of the product carrier and then lowered to a plate where it solidifies into small pieces. Factors such as melt viscosity and pipette size can influence particle size and shape. The fall solution does not use organic solvents and thus has no solvent evaporation issues. This approach often avoids the pulverization, sifting, and compressibility difficulties.

\section{9] CO-PRECIPITATION TECHNIQUE}

Co-precipitation is a known technique that improves the degradation of products that are poorly water-soluble, thereby increasing bioavailability. Drop wise to the drug and carrier solution under constant stirring, non solvent is added in this method. In the non-solvent addition process, the substance and the carrier are co-precipitated to form microparticles. The subsequent deposition of the microparticles is subsequently filtered and dried. Mixed the appropriate quantity of polymer and drug, then added solvent to get a clear solution. The Solution was first dried under vacuum at room temperature, and kept in the incubator $\left(37^{\circ} \mathrm{C}\right)$ for 12 hours. Finally, this was replaced by sieves.

\section{0] USE OF CO -SURFACTANTS}

The utility of the Solvency surfactant systems is well known. Surfactant adsorption on solid surfaces can change its hydrophobicity, surface charging, and other key properties that control interfacial processes.

Types include flocculation / dispersion, floating, wetting, solubilization, detergence, improved oil recovery and inhibition of corrosion. Surfactants were also reported to induce solvation / plasticisation, resulting in reduced melting of active pharmaceutical ingredients, glass transition temperature, and combined glass transition temperature for solid dispersion. Because of these peculiar properties, the investigators stimulated interest in the preparation of strong dispersions by surfactants [31, 32].

Recently a new class of surfactants, geluciers with different melting points and HLB values (hydrophilic and lipophilic balance) is suggested. Gelucire excipients have been used in preparation of semi-solid dispersions. They are solid waxy materials which have an amphiphilic character. Gelucires are the polyglycolized saturated glycerides consisting of mono-, di-, and tri-glycerides, and mono- and trans-fatty acid esters of polyethylene glycol. The type and proportion of the individual variable is unique to a given degree of gelucire. Geluciers with low HLB may be used for rapid release to reduce the risk of product degradation and higher HLBs. The two are Gelucire 44/14 and 50/13 Examples of this synthetic group where 44 and 50 represent the melting point, while 14 and 313 
represent the HLB gelucire values 6162.Stable dispersions were tested with the antiviral agent uc-781-polyethylene glycol 6000-gelucire 44/14 and UC-781- PEG 6000-gelucire 44/14- PVP k 30. Solubility improved, dissolution and stability increased.

\section{CONCLUSION}

Drug solubility in aqueous media is a key factor that strongly influences their dissolution rate and bioavailability after oral administration, leading to low bioavailability. Improving the solubility of these drugs is still one of the toughest aspects of drug development. Over the years a range of tools to improve drug solubility and drug dissolution have been developed. Strong dispersion process is one of the effective approaches to attaining the goal of improving solubility of poorly water soluble drugs. Different techniques, as defined in this report, are used effectively to prepare solid dispersions in the bench and lab scale and can also be used on an industrial scale.

\section{REFERENCES}

[1] Hecq J, Deleers M, Fanara D, Vranckx $H$ and Amighi $K$. Preparation and characterization of nanaocrystals for solubility and dissolution rate enhancement of nifidipine. Int. J. Pharm. 299, 2005, 167-177.
[2] Kapsi S G and Ayres J W. Processing factors in development of solid solution formulation of itraconazole for enhancement of drug dissolution and bioavailability. Int. J. Pharm. 299, 2001, 193-203.

[3] Orienti I, Bigucci F, Luppi B, Cerchiara T, Zuccari G, Giunchedi $\mathrm{P}$ and Zecchi V. Polyvinylalcohol substituted with triethylleneglycolmonoethylether as a new material for preparation of solid disper- sions of hydrophobic drugs. Int. J. Pharm. 54, 2002, 229-233.

[4] Patro S, Himasankar K, Choudhury A A and Rao M E B. Effect of some hydrophilic polymers on dissolution rate of roxitromycin. Indian $\mathrm{J}$. Pharm. Sci. 67(3), 2005, 334-341.

[5] Omaima A S, Mohammed A H, Nagia $\mathrm{A} M$ and Ahmed $\mathrm{S} Z$. Formulation and optimization of mouth dissolve tablets containing rofecoxib solid dispersions. AAPS PharmSciTech 7(2), 2006, E1- E9. Takano, Niichiro, Kawashima, Hiroyuki, Shinoda, Yasuo, Inagi and Toshio. Solid dispersion compositions. United States Patent No. 6753330, 2004.

[6] Yiyun C, Tongwen X and Rongqiang F. Polyamidoamine dendrimers used as solubility 
enhances of ketoprofen. Eur. J. Med. Chem. 40, 2005,1390-1393.

[7] Mohan Babu G V M, Prasad D S, and Ramana Murthy $\mathrm{K}$ V. Evaluation of modified gum karaya as carrier for the dissolution enhancement of poorly watersoluble drug Nimodipine. Int. J. Pharm. 234, 2002, 1-17.

[8] Gibaldi M. Ed., In; Biopharmaceutics and clinical pharmacokinetics, $4^{\text {th }}$ Edn, Pharma Book Syndi- cate, Hyderabad, 2005, 48

[9] Tanaka N, Imai K, Okimoto K, Ueda S, Rinta Ibuki Y T, Higaki K and Kimura T. Development of novel sustained-release system, disinmtegration-controlled matrix tablet (DCMT) with solid dispersion granules of nilcadipine (II): In vivo evaluation. Journal of controlled release 122, 2006, 5156.

[10] Duncan Q M C. The mechanism of drug release from solid dispersions in water-soluble polymers. Int. J. Pharm. 231, 2002, 131-144.

[11] Vasconcelos $\mathrm{T}$, et al., Solid dispersions as strategy to improve oral bioavailability of poor water soluble drugs. J Pharma Sci. 2007; 12: 23-24.
[12] Sonia Dhiman, et al., Solid Dispersions: Opportunity in Drug Delivery System, 2012; 4(10): 478-486.

[13] Tagalpallewar, V. R. et al., Enhancement of Solubility of poorly water soluble drug by solid dispersion technique, 2015; 6(2): 15.

[14] Zerrouk N, Chemtob C, Arnaud P, Toscani $\mathrm{S}$ and Dugue J. In vitro and in vivo evaluation of carbemazepine-PEG 6000 solid dispersions. Int. J. Pharm. 225, 2001, 49-62.

[15] Boral A, Sen N, Ghosh L K and Gupta B K. Solid dispersion technology for controlling drug release and absorption. The eastern pharmacist, April 1995, 141-143.

[16] Hasegawa S, Hamaura $T$, Furuyama N, Kusai A, Yonemochi $\mathrm{E}$ and Terada K. Effects of water contents in physical mixture and heating temperature on crystallinity of troglitazone- PVP K 30 solid dispersions prepared by closed melting method. Int. J. Pharm. 302, 2005, 103-112.

[17] Nakano, Minoru, Uemura, Toshinobu, Morizane, Shinichi, Okuda, Kiyoshi, Nakata and Keiko. Method of producing a 
solid dispersion of a sparingly water-soluble drug, nilvadipine. United State Patent No. 5340591, 1994

[18] A. K. Chaturvedi, et al., Solubility Enhancement of Poorly Water Soluble drugs by Solid Dispersion, 2012; 3(1): 26-34.

[19] Rahul M. Patil, et al., Solid Dispersion: Strategy to Enhance Solubility, 2011; 8(2):11.

[20] Satish K. Patil, Strategies for Solubility Enhancement of Poorly Soluble drugs, 2011; 8(2): 20

[21] Chronakisa I S, Triantafyllou A O, $\mathrm{R} \mathrm{O}$ " ste. Solidstate characteristics and redispersible properties of powders formed by spray-drying and freeze-drying cereal dispersions of varying (1 3, 14$)-\beta$ -glucan content. Journal of cereal science 40, 2005, 183-193.

[22] Patrice $T$ T, Stephanie B and Hatem F. Preparation of redispersible dry nanocapsules by means of spray drying: Development and characterization. Eur. J. Pharm. Sci. 30, 2007, 124 135.

[23] Rankell A S, Lieberman H A and Schiffmann R F. Drying. In: L. Lachman, H.A. Lieberman and J.L.Kanig eds. The theory and practice of industrial pharmacy. 3rd edition, Varghese Publishing house, Bombay, 1987, pp no 61.

[24] Weuts I, Kempen D, Verreck G, Decorte A, Heymans K, Peeters J, Brewster M and Mooter G V den. Study of the physicochemical properties and stability of solid dispersions of loperamide and PEG 6000 prepared by spray drying. Eur. J. Pharm. Biopharm. 59, 2005, 119-126.

[25] Chauhan B, Shimpi S and Paradkar A. Preparation and evaluation of glibenclamidepolyglycolized glycerides solid dispersions with silicon dioxide by spray drying technique. Eur. J. Pharm. Sci. 26, 2005, 219-230.

[26] Ueno Y, Yonemochi E, Tozuka Y, Yamamura S, Oguchi $\mathrm{T}$ and Yamamoto K. Characterization of amorphous ursodeoxycholic acid prepared by spray-drying. J. Pharm. Pharmacol. 50(11), 1998 Nov, 1213-1219

[27] Chen R, Tagawa M, Hoshi N, Ogura T, Okamoto $\mathrm{H}$ and Danjo $\mathrm{K}$. Improved dissolution of an insoluble drug using a 4-fluid nozzle spray-drying technique. Chem. Pharm. Bull. 52(9), 2004 Sep, 1066-1070.

[28] Tanno, Fumie, Nishiyama and Yuichi. Process for producing a 
pharmaceutical solid preparation containing a poorly soluble drug. United states patent No. 6872336.

[29] Takeuchi H, Nagira S, Yamamoto $\mathrm{H}$ and Kawashima Y. Solid dispersion particles of amorphous indomethacin with fine porous silica particles by using spray-drying method. Int. J. Pharm. 293, 2005, 155164.

[30] Takeuchi H, Nagira S, Yamamoto $\mathrm{H}$ and Kawashima Y. Solid dispersion particles of tolbutamide prepared with fine silica particles by the spray-drying method. Powder technology 141, 2004, 187-195.

[31] Ghebremeskel A N, Vemavarapu $C$ and Lodaya M. Use of surfactants as plasticizers in preparing solid dispersions of poorly soluble API: Selection of polymer surfactant combinations using solubility parameters and testing the processability. Int. J. Pharm. 328, 2007, 119129.

[32] Zhang $R$ and Somasundaran $P$. Advances in ad- sorption of surfactants and their mixtures at solid/solution interfaces. Advances in colloid and interface science 123-126, 2006, 213-229.

[33] Damian F, Blaton N, Naesens L, Balzarini J, Kinget R, Augustijns
P and Mooter G Van den. Physicochemical characterization of solid dispersions of the antiviral agent UC-781 with polyethylene glycol 6000 and Gelucire 44/14. Eur. J. Pharm. Sci. 10, 2000, 311322.

[34] Damian F, Blaton N, Kinget R and Mooter G Van den. Physical stability of solid dispersions of the antiviral agent UC-781 with PEG 6000, Gelucire 44/14 and PVP K3. Int. J. Pharm. 244, 2002, 87- 98. 